\title{
The burden and management of self-inflicted injuries at a tertiary care hospital in Mwanza, Tanzania
}

\author{
ALPHONCE B. CHANDIKA ${ }^{1 *}$, PHILLIPO L. CHALYA', KIYETI A. HAULI², PETER F. RAMBAU3,4, ADELA A. \\ MWAKANYAMALE ${ }^{5}$ and JAPHET M. GILYOMA ${ }^{1}$ \\ 'Department of Surgery, Catholic University of Health and Allied Sciences- Bugando, Mwanza, Tanzania \\ ${ }^{2}$ Department of Psychiatry, Catholic University of Health and Allied Sciences- Bugando, Mwanza, Tanzania \\ ${ }^{3}$ Department of Pathology, Catholic University of Health and Allied Sciences-Bugando, Mwanza, Tanzania \\ ${ }^{4}$ Forensic Medicine unit, Bugando Medical Centre, Mwanza, Tanzania \\ ${ }^{5}$ Department of Community, Faculty of Nursing, Muhimbili University of Health and Allied Sciences, Dar es \\ Salaam, Tanzania
}

\begin{abstract}
Background: Self-inflicted injuries are a serious but neglected public health problem in developing countries and contribute significantly to the global injury burden. There is a paucity of published data regarding self-inflicted injuries in Tanzania. This study describes the aetiology, patterns and treatment outcome of these injuries in Mwanza, Tanzania.

Methods: This was a combined retrospective and prospective study of self-inflicted injury patients who were managed at Bugando Medical Centre in Mwanza between February 2007 and April 2014. Data were collected using a pretested and coded questionnaire and analysed using SPSS computer software version 17.0

Results: A total of 136 patients ( $M: F=3.3: 1)$ were enrolled into the study. The median age was 30 years. Psychiatric illness (30.9\%) was the most common risk factor for self-inflicted injuries. Self-poisoning (35.3\%) was the most frequent mechanism of self-inflicted injuries. This was followed by cutting/stabbing and jumping from heights in $23.5 \%$ and $20.6 \%$ of patients, respectively. The trunk was the most frequent (31.9\%) body region affected. Open wounds were the most (4.2\%) common type of injuries sustained. The majority (72.1\%) of patients were treated surgically. The complication rate was $50.7 \%$ and it was significantly associated with delayed presentation $(p=0.012)$. The median hospital stay was 12 days. Patients with long bone fractures and those who had surgical site infection stayed longer in the hospital $(p<0.001)$. The mortality rate was $22.8 \%$. Late presentation ( $>24$ hour), severe injuries (KTS II $\leq 6$ ) and presence of surgical site infections were the main predictors of mortality $(p<0.001)$.

Conclusion: Self-inflicted injuries are an emerging but neglected public health problem in Mwanza, Tanzania and contribute to unacceptably high morbidity and mortality. Addressing the root causes of selfinflicted injuries may reduce the incidence of these injuries in our environment.
\end{abstract}

Keywords: Self-inflicted injuries, aetiology, patterns, treatment outcome, Tanzania

\section{Introduction}

Self-inflicted injuries, defined as injuries inflicted on oneself with or without suicidal intent, are a serious but neglected public health problem and carry considerable mortality and morbidity among otherwise fit young individuals (Greydanus \& Shek, 2009). Self-inflicted death accounts for $1.5 \%$ of all deaths and is the tenth leading cause of death worldwide (Greydanus \& Shek, 2009; Karch et al., 2010). Injuries resulting from self-harm have a profound social, emotional, and economic impact on those affected, including family, friends and the community. Self-inflicted injuries are costly as their emergency treatment, hospitalization and long term care divert scarce resources from other health care priorities (Corso et al., 2007; Greydanus \& Shek, 2009; Karch et al., 2010). In Sub-Saharan countries, more than 80 percent of victims of intentional trauma including self-inflicted injuries have no definable source of private or governmental health care

\footnotetext{
* Correspondence E-mail: achandika2001@yahoo.com
} 
insurance at the time of their injury (Moshiro et al., 2005; Kobusingye, 2008; Demyttenaere et al., 2009).

Globally, the reported incidence of self-inflicted injuries varies considerably from country to country and centre to centre. These account for 3-5\% of all intentional injuries in high-income countries such as the United States (Corsoet al., 2007). However, in developing countries like Tanzania, the incidence is increasing at a high rate partly because of increasing conflict over limited resources, poor socioeconomic status, poverty, unemployment, easy access to firearms, alcohol and substance misuse and increased crime rates (Kobusingye, 2008). Self-harm individuals suffer from a wide variety of problems, including psychiatric disorders, unemployment, financial difficulties, substance abuse, a difficult childhood, poor physical health, family and intimate partner violence, and relationship difficulties (Dyck et al., 1988; Suominen et al., 1996; Goldney, 2002). Moreover, these individuals are also at high risk for future self-harming and suicidal behaviour (Suominen et al., 1996).

Methods used to self-inflict injury have been reported to vary according to gender. Whereas self-poisoning accounts for the majority of self-inflicted injuries in females, males are more likely to use more lethal methods, such as firearms, hanging, jumping from heights or poisoning by gas (Doshi et al., 2005). Males are also more likely to use other methods which include extremes of heat or cold, electrocution and suicidal behaviour involving a moving object or vehicle (Doshi et al., 2005).

The management of self-inflicted injuries poses a great challenge and requires a multidisciplinary approach requiring the close collaboration of the surgeon, the anaesthetist and the psychiatrist (Duncan, 1975; Ladapo, 1979; Bailey, 1997; Bhattacharjee et al., 1997; Herzog et al., 2005). The surgeon assesses the injury and repairs the severed tissues with the aim of restoration anatomical function. The anaesthetist secures an uncompromised airway and makes sure the patient is breathing; the psychiatrist provides adequate care and supervision during and after surgical treatment (Bailey, 1997; Bhattacharjee et al., 1997; Herzog et al., 2005; Manilal et al., 2011). However, in most developing countries such as Tanzania, late presentation to health facilities coupled with lack of advanced pre-hospital and ineffective ambulance system for transportation of patients to hospital care contributes significantly to increasing morbidity and mortality.

Although there is growing interest in research on self-inflicted injuries, use of health services by individuals who harm themselves, the economic and social impact of self-harm and risk factors, many of the published reports are from high-income countries (Marbella et al., 2005; Lopez et al., 2006; Mathers \& Loncar, 2006; Mathers et al., 2009). There is a paucity of information on self-inflicted injuries in most low-income countries where greater emphasis has been placed on injuries related to road traffic accidents, which are more common (Gilyoma \& Chalya, 2014). This study was conducted in Mwanza, north-western Tanzania to describe the experience in the management of self-inflicted injuries, outlining the aetiology, patterns and treatment outcome of these injuries with the hope that our findings will be a guide to offer preventive and therapeutic measures in these patients and ultimately improve their outcome.

\section{Materials and Methods}

\section{Study design and setting}

Between February 2007 and April 2014, a combined retrospective and prospective study of selfinflicted injury patients was conducted at the Accident and Emergency of Bugando Medical Centre (BMC). Bugando Medical Centre is a 890-bed, tertiary care and teaching hospital for the Catholic University of Health and Allied Sciences-Bugando, Mwanza, Tanzania (CUHAS). It is located in Mwanza city, along the shores of Lake Victoria in north-western Tanzania. The hospital provides service to a population of approximately 13 million people from its neighbouring regions 
in north-western Tanzania. The hospital receives all trauma patients requiring expertise management as there is no trauma centre or established advanced pre-hospital care for trauma patients in Mwanza city.

\section{Study population}

All patients who presented with self-inflicted injury during the study period were included in the study. Patients who presented in a "shocked" state (i.e. systolic blood pressure $<90 \mathrm{mmHg}$ ) and those who were under 18 years of age, their parents, guardian or relatives had to consent on their behalf. Patients with incomplete data and those who were brought in dead were excluded from the study. The details of patients who presented from February 2007 to July 2009 were retrieved retrospectively from patient registers kept in the Medical Record Departments, the surgical wards, and operating theatre. Patients who presented to the Accidents and Emergency (A\&E) department between August 2009 and April 2014 were prospectively enrolled in the study after signing an informed written consent for the study. All patients who were recruited prospectively were, before enrolled in the study, resuscitated in the A\&E department according to Advanced Trauma Life Support (ATLS). From the A \& E department, patients were taken to theatre for surgical intervention and from there, patients were taken into the surgical wards or the intensive care unit (ICU) for admission.

\section{Data collection}

The severity of injury was determined using the Kampala Trauma Score II (KTS II) (Mutooro et al., 2010). KTS II is scored based on the following parameters (A) age in years (i.e. $5-55=1,\langle 5$ and $>$ $55=0$ ), (B) number of serious injuries (i.e. none $=2$, one injury $=1$ and $>$ one injury $=0$ ), (C) systolic blood pressure (i.e. $>89 \mathrm{mmHg}=2,89-50 \mathrm{mmHg}=1, \leq 49 \mathrm{mmHg}$ ), (D) respiratory rate (i.e. 0 $29 / \mathrm{min}=2,>30 / \mathrm{min}=1$ and $\leq 29 / \mathrm{min}$ ) and neurologic status (i.e. alert $=3$, responds to verbal stimuli $=2$, responds to painful stimuli $=1$ and unresponsive $=0$ ) on presentation. Kampala Trauma Score total $=A+B+C+D+E$. Severe injury consists of a KTS $I I \leq 6$, moderate injury $7-8$, and mild injury 9-10. Patients with head injuries were classified according to Glasgow Coma Scale (GCS) into: severe (GCS 3-8), moderate (GCS 9-12) and mild (GCS 13-15). Routine investigations including haematological (haemoglobin, blood grouping and cross-matching), biochemical (serum creatinine and serum electrolytes) and imaging (plain x-rays of the chest / abdomen, pelvis, spines and limbs, abdominal ultrasound and CT scan) were performed on admission. Depending on the type of injury, patients were treated either conservatively or surgically.

All the data regarding study population were collected and compiled in a structured questionnaire with thoroughly looked upon ethical implication. All the data pertinent to the patient were kept confidential. Data were categorized according to the demographic pattern of the patient, cause and motivating factors behind the injury, pre-hospital care, body site injured, type and the extent of the tissue damage or involved, presentation during admission, time taken or delay from the incidence to the hospital attendance (injury-arrival interval) and duration of the hospital stay, type of special intervention given, records of mortality, noticeable morbidity and outcome. Patients who were prospectively enrolled in the study were followed up till death or discharge and thereafter for up to 12 months after surgery.

\section{Data analysis}

Statistical data analysis was done using SPSS software (Statistical Package for the Social Sciences, version 17.0, SPSS Inc, Chicago, III, USA). The median (+ IQR) and ranges were calculated for continuous variables whereas proportions and frequency tables were used to summarize categorical variables. Chi-square $\left(\chi_{2}\right)$ test were used to test for the significance of association between the independent (predictor) and outcome (dependent) variables. The level of significance was considered as $p$-value $<0.05$. Multivariate logistic regression analysis was 
used to determine predictor variables that predict the treatment complications, hospital stay and mortality

\section{Ethical considerations}

The study was carried out after the approval by the department of surgery and BMC/CUHASBugando ethics review board. An informed written consent was obtained from patients/relatives who were recruited prospectively. The need for consent for participants who were recruited retrospectively was deemed unnecessary according to national regulations.

\section{Results}

During the period of study, a total of 152 patients presented to BMC with self-inflicted injuries. Out of these, 16 patients were excluded from the study due to failure to meet the inclusion criteria and incomplete data. Thus, 136 patients were enrolled into the study. Of these, $24(17.6 \%)$ patients were studied retrospectively and the remaining $112(82.4 \%)$ patients were studied prospectively. The age of patients at presentation ranged from 9 to 76 years with a median of 30 years (interquartile range, 24 to 43 years). The modal age group was 21-30 years accounting for $40.4 \%$ of cases. Out of 136 patients, 104 (76.5\%) were males and 32 (23.5\%) were females with a male to female ratio of 3.3:1. The vast majority of patients, $98(72.1 \%)$ had primary or no formal education and most of them, 122 (89.7\%) had no employment. The majority of patients came from within areas of Mwanza City with 125 (91.9\%) victims coming from low-income areas (high density areas) and the remaining 11 (8.1\%) victims came from high-income areas (low density areas). Only nine (6.6\%) patients had definable source of private or governmental health care insurance at the time of their injury. Previous history of self-harm was reported in 8 (2.9\%) patients. Out of the 136 patients, 60 (44.1\%) had associated co-morbid illness namely: psychiatric illness (including depression and other mental illnesses) in 42 (70.0\%) patients, hypertension in $6(10.0 \%)$, malignancies in $5(8.3 \%)$, Diabetes mellitus in $4(6.7 \%)$, HIV infection in $2(3.3 \%)$ and tuberculosis in 1 $(1.7 \%)$ patient. Self-poisoning (mainly drug overdose and other chemical agents unknown to patients) was the most frequent mechanism of self-inflicted injuries accounting for $35.3 \%$ of cases (Table 1).

Table 1: Mechanism of Self-Inflicted Injury versus $\operatorname{sex}(\mathrm{N}=136)$

\begin{tabular}{llll}
\hline & Sex & & \\
\cline { 2 - 3 } Mechanism of self-inflicted injury & Male $(\mathbf{N} / \%)$ & Female $(\mathbf{N} / \%)$ & Total (N/\%) \\
\hline Self-poisoning & $22(16.2)$ & $26(19.1)$ & $48(35.3)$ \\
Cutting/stabbing & $32(23.5)$ & 0 & $32(23.5)$ \\
Jumping from heights & $27(19.9)$ & $1(0.7)$ & $28(20.6)$ \\
Hanging & $10(7.4)$ & 0 & $10(7.4)$ \\
Firearms/ guns & $6(4.4)$ & 0 & $6(4.4)$ \\
Drowning & $4(2.9)$ & 0 & $4(2.9)$ \\
Others & $3(2.2)$ & $5(3.7)$ & $8(5.9)$ \\
Total & $104(76.5)$ & $32(23.5)$ & $136(100)$ \\
\hline
\end{tabular}

Self-poisoning was more common in males than in females though this association was not statistically significant $(p=0.675)$. Psychiatric illness was the most common risk factor for selfinflicted injuries accounting for $30.9 \%$ of cases (Figure 1 ).

The vast majority of patients, 96 (70.6\%) reported to the A \& E department within 24 hours after injury. None of the patients received any pre-hospital care and most of them 112 (82.4\%) were brought in by relatives, friends or Good Samaritans while 24 (17.6\%) were brought by police. No patient was brought in by an ambulance. The waiting time (i.e. time interval taken 
from reception at the $A \& E$ department and reception of treatment) ranged from 30 minutes to 12 hours with a median of 6 hours. The majority of patients, 106 (77.9\%) were attended to within 2-6 hours of arrival to the A \& E department.

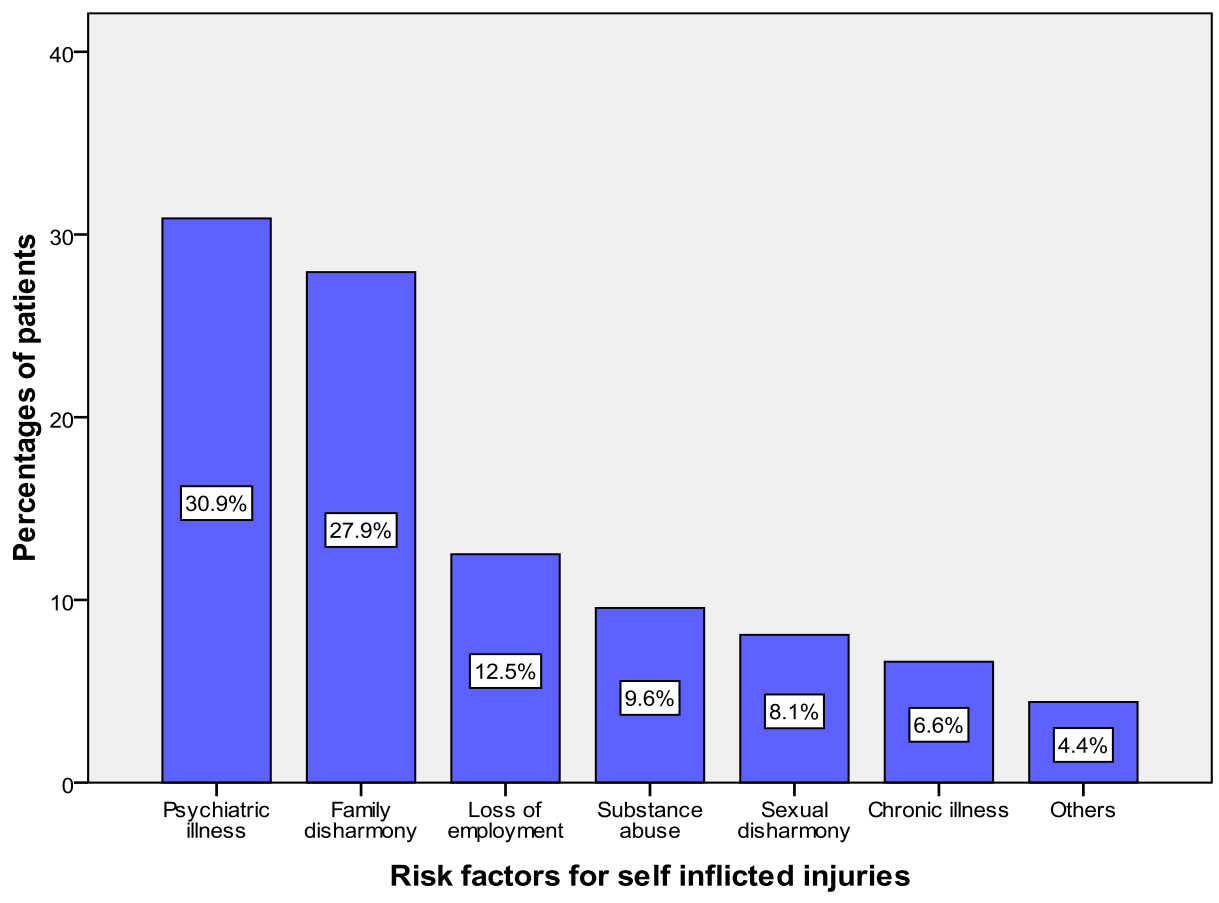

Figure 1: Distribution of patients according to motivating (risk) factors for self-inflicted injuries

The trunk (chest and abdomen) was the most frequently affected body region in $31.9 \%$ of cases. Lesions on the trunk were more common on the anterior aspect than on the posterior aspect. Musculoskeletal system (limbs) 34 (23.6\%), was the second most frequently affected body region. Other affected regions of the body included head/neck (15.3\%), pelvis (10.4\%), spines (6.9\%), genitalia (4.2\%0 and others (7.6\%). Isolated injuries occurred in $80(58.8 \%)$ patients while $56(41.2 \%)$ patients had multiple injuries. Open wounds (i.e. bruises, abrasions, lacerations, crush wounds) were the most common type of injuries sustained in $41.2 \%$ of patients (Table 2 ).

Table 2: Distribution of patients according to the type of injury

\begin{tabular}{lll}
\hline Type of injury & Frequency & Percentages \\
\hline Open wounds & 56 & 41.2 \\
Fractures & 26 & 19.1 \\
Visceral injuries & 22 & 16.2 \\
Pneumothorax & 16 & 11.8 \\
Haemothorax & 15 & 11.0 \\
Cut genitalia (penis) & 6 & 4.4 \\
Others & 12 & 8.8 \\
\hline
\end{tabular}

With reference to Kampala Trauma Score II, out of the 112 patients studied prospectively, 66 (58.9\%) had moderate injuries, 24 (21.4\%) had mild injuries and $22(19.6 \%)$ had severe injuries. Out of 22 patients who had head injuries, the Glasgow coma scale indicated that most of the patients, $12(54.5 \%)$ sustained mild head injury, $6(27.3 \%)$ sustained moderate head injury and $4(18.2 \%)$ had severe head injury. 
A total of $98(72.1 \%)$ patients underwent 166 surgical procedures and the remaining 38 (27.9\%) were treated by non-operative approach (conservatively) with intravenous fluids, analgesics, antibiotics, tetanus toxoid prophylaxis, blood transfusion, wound dressing etc. Surgical debridement was the most frequent surgical procedure performed (Figure 2). All patients were subjected to psychiatric assessment after definitive treatment.

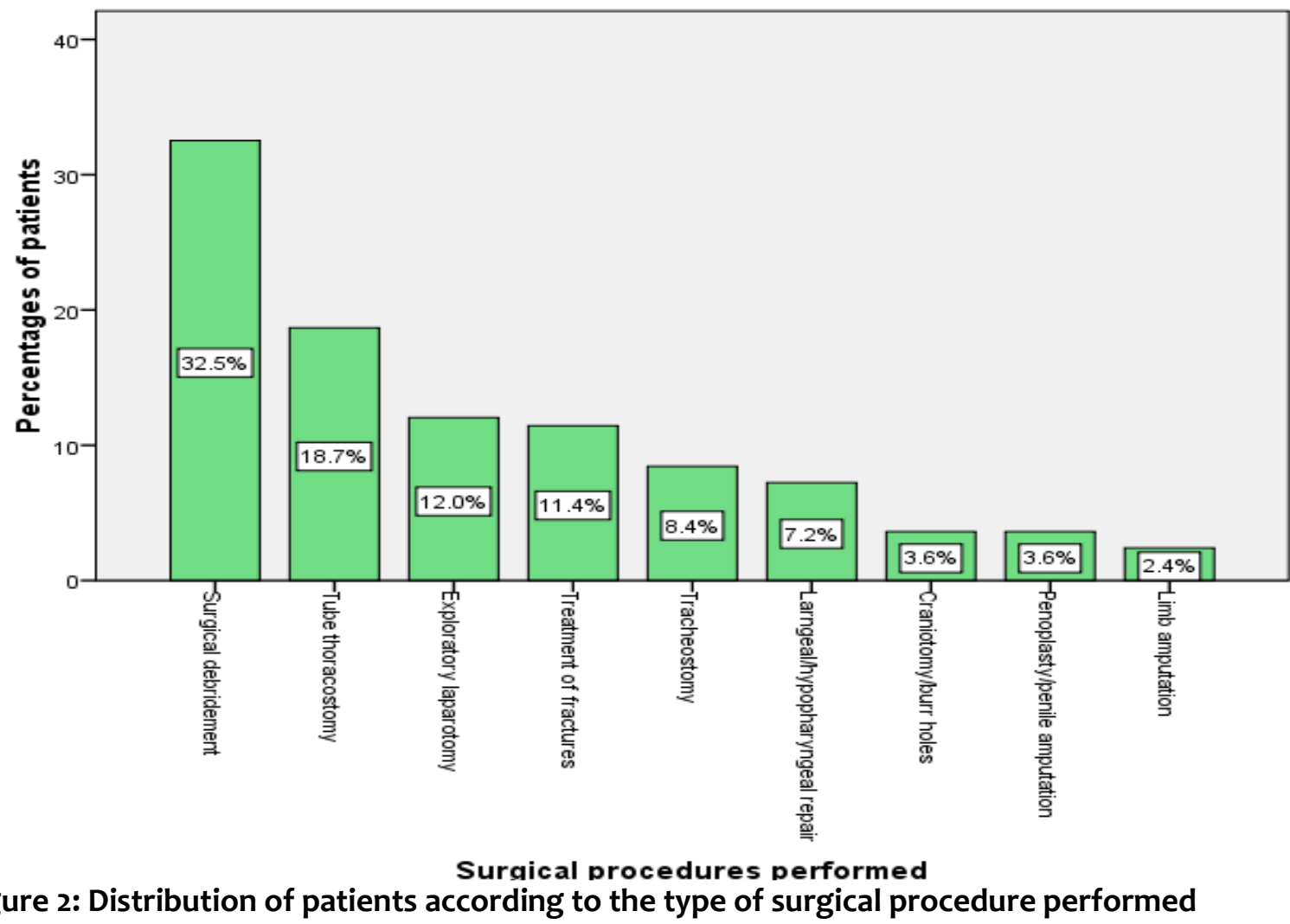

Sixty-nine (50.7\%) patients developed 74 complications of which surgical site infections (32.4\%; $\mathrm{n}=24$ ) were the most common complications (Table 3).

Table 3: Distribution of patients according to postoperative complications

\begin{tabular}{lll}
\hline Post-operative complications & Frequency & Percentages \\
\hline Surgical site infections & 24 & 32.4 \\
Complications of fracture treatment & 6 & 8.1 \\
Wound dehiscence & 6 & 8.1 \\
Enterocutaneous fistula & 5 & 6.8 \\
Secondary peritonitis & 5 & 6.8 \\
Aspiration pneumonia & 4 & 5.4 \\
Laryngeal'/pharyngeal stenosis & 4 & 5.4 \\
Permanent tracheostomy & 3 & 4.1 \\
Urethral structure & 2.7 \\
Urinary tract infection & 2 & 2.7 \\
Empyema thoracis & 2 & 2.7 \\
Keloid formation & 2 & 2.7 \\
Others & 2 & 12.2 \\
\hline
\end{tabular}

Complication rate was significantly associated with delayed presentation $(\mathrm{p}=0.012)$. Thirty-one patients died giving a mortality rate of $22.8 \%$. Late presentation ( $>24$ hours) $(\mathrm{OR}=3.6,95 \% \mathrm{Cl}(1.4-$ 6.7), $\mathrm{p}=0.011)$, severe injuries $(\mathrm{OR}=1.2,95 \% \mathrm{Cl}(1.0-4.5), \mathrm{p}=0.014)$ and presence of surgical site 
infections $(\mathrm{OR}=10.2,95 \% \mathrm{Cl}(9.3-13.5), p=0.001)$ were the main predictors of mortality on multivariate logistic regression analysis.

The overall length of hospital stay (LOS) ranged from 1 to 56 days with a median of 12 days (IQR of 10 to 14 days). The median LOS for non-survivors was 4 days (IQR of 2 to 6 days). The majority of patients (survivors), 72 (68.6\%) stayed in hospital for less than a two-week duration. Patients who had associated long bone fractures and those who had surgical site infections stayed significantly longer in the hospital $(p<0.001)$.

Out of the 105 survivors, 96 (91.1\%) patients were discharged well, 7 (6.7\%) patients were discharged against medical advice and the remaining $2(1.9 \%)$ patients were discharged with permanent disabilities related to permanent tracheostomy. After discharge, all patients who were well at discharge were discharged through Psychiatric outpatient clinic for proper psychiatric evaluation. Of the survivors, only 45 (42.9\%) patients were available for follow-up at 6-12 months and the remaining $60(57.1 \%)$ patients were lost to follow-up.

\section{Discussion}

Self-inflicted injuries constitute a growing but neglected epidemic in developing countries and contribute significantly to the global injury burden (Goldney, 2002; Lopez et al., 2006; Greydanus \& Shek, 2009). In this study, most of patients were young in their third decade of life and were males, who are still in their reproductive and economically productive period of life. This finding agrees with studies elsewhere (Bhattacharjee et al., 1997; Manilal et al., 2011; Gilyoma \& Chalya, 2014) but in disagreement with data from China which shows female predominance (Dyck et al., 1988). Male predominance in this age group may be attributable to their active participation in risk taking behaviours and their frequent involvement in use of substance abuse (Bhattacharjee et al., 1997). In keeping with other studies (Platt, 1984; Bhattacharjee et al., 1997; Manilal et al., 2011; Gilyoma \& Chalya, 2014), most of patients in the present study were unemployed and uneducated and the majority of them came from low-income areas of the city and only few had definable source of health care insurance at the time of their injury. This observation has an implication on accessibility to health care facilities. Unemployment can act as a stressful life event leading to suicide (Platt, 1984) with studies suggesting an increase in the para-suicide and suicide rates among unemployed individuals than in the general population (Shah \& Bhandarkar, 2008).

Previous studies regarding self-inflicted injuries have shown that a history of a previous self-inflicted injury is a key risk factor for a repeated self-inflicted injury (Dyck et al., 1988; Doshi et al., 2005; Gilyoma \& Chalya., 2014). The finding that previous history of self-harm was reported in a number of patients concurs with other studies elsewhere (Bailey, 1997; Manilal et al., 2011; Gilyoma \& Chalya, 2014) reflecting that individuals with self-inflict injuries are predisposed to future self-harming and suicidal behaviour. In this study, associated medical co-morbidities were reported in $44.1 \%$ of cases. Of these, psychiatric illness accounted for seventy percent of cases. This observation agrees with other studies elsewhere (Dyck et al., 1988; Suominen et al., 1996; Karch et al., 2010). As found in our study, psychiatric illness has been reported to be associated with self-harm (Dyck et al., 1988; Suominen et al., 1996) and are the strongest predictors of selfinflicted injuries (Suominenet al., 1996). Chronic and terminal illnesses such as cancer and HIV infection have also been reported to be a contributing factor for self-inflicted injuries (Suominen et al., 1996; Greydanus \& Shek, 2009). In the current study, psychiatric illness was the most common risk factor for self-inflicted injuries. Similar observations have been reported elsewhere (Dyck et al., 1988; Suominen et al., 1996; Greydanus \& Shek, 2009). Other risk factors for selfinflicted injuries reported in literature include poverty, unemployment, financial difficulties, substance abuse, terminal illness, a difficult childhood, poor physical health, family and intimate partner violence, and relationship difficulties individuals (Greydanus \& Shek, 2009). 
In agreement with other studies (Reith et al., 2003; Olfson et al., 2005), self-poisoning was the most frequent mechanism of self-inflicted injuries in this study and commonly occurred among males than females. Studies have shown that by comparison, females prefer less violent means and they are more likely to end their life through the use of poisoning, whereas males are more likely to use violent methods such as hanging, cutting or piercing, jumping suffocation or through use of firearms (Reith et al., 2003). This observation agrees with our study where the use of poisoning to self-harm was more common in females and the other methods such as cutting or piercing, hanging, jumping and use of firearms were more common among males.

The prehospital care of trauma patient has been reported to be the most important factor in determining the ultimate outcome after the injury (Spicer \& Miller, 2000; Norberg, 2000; Vajani et al., 2007; Adoga et al., 2010). However, as reported from other studies in developing countries (Spicer \& Miller, 2000; Gilyoma \& Chalya, 2014), none of our patients had pre-hospital care. The lack of advanced pre-hospital care and ineffective ambulance system for transportation of patients to hospitals are a major challenges in providing care for trauma patients in our environment and have contributed significantly to poor outcome of these patients.

This study showed that mostly the trunk was the most frequent body region affected and distributions of lesions were more common on the anterior aspect than on the posterior aspect. Similar anatomical site distribution of the injury was reported by others (Norberg, 2000; Olfson et al., 2005). Most of patients in this study sustained soft tissue injuries. This is in agreement with findings from other studies (Spicer \& Miller, 2000; Norberg, 2000; Vajani et al., 2007). A good knowledge of the nature and type injury allows the clinicians to understand the type of weapon used and this is of great importance for medico-legal purposes and surgical treatment. Most of our patients were treated surgically, which is in agreement with other similar studies (Bhattacharjee et al., 1997; Herzog et al., 2005; Gilyoma \& Chalya., 2014). As reported by other studies (Bhattacharjee et al., 1997; Herzog et al., 2005; Gilyoma \& Chalya., 2014), surgical debridement was the most common surgical procedures performed in this study. In this study, all patients were considered for the psychiatric consultation. This was because the act of self-harm is a sign of underlying mental illness and there is possibility of a subsequent attempt (Dyck et al., 1988; Suominen et al., 1996). In keeping with other studies (Olfson et al., 2005; Adoga et al., 2010; Gilyoma \& Chalya., 2014), more than 50\% of patients developed complications of which surgical site infections was the most common complications. High rate of surgical site infection reflecting that early and meticulous wound care is of great importance in the management of self-inflicted injuries. Complication rate was significantly associated with delayed presentation. Early recognition and management of complications following self-inflicted injury is of paramount in reducing the morbidity and mortality resulting from these injuries.

The length of hospital stay has been reported to be an important measure of morbidity among trauma patients. Prolonged hospitalization is associated with an unacceptable burden on resources for health and undermines the productive capacity of the population through time lost during hospitalization and disability (Norberg, 2000). The median duration of hospital stay in this study was found to be longer in patients who had associated long bone fractures and those who had surgical site infections. Our mortality rate in this study was higher than that reported by others (Bhattacharjeeet al., 1997; Gilyoma \& Chalya., 2014). Late presentation, severe injuries and surgical site infections were the major factors responsible for the high mortality in our patients. Addressing these factors is mandatory to be able to reduce mortality associated with these injuries. Generally, the overall outcome of our patients was good as more than ninety percent of patients (survivors) were discharged well without permanent disabilities.

The potential limitation of this study is the fact that information about some patients was incomplete in view of the retrospective nature of the study. This might have introduced some bias in our findings. Another potential limitation was that the analysed group of patients was treated at a single medical centre. For that reason, the results may not represent the situation in 
other parts of Tanzania. The history of psychiatric illness and use of substance abuse was based on self-reporting from either the patient or relatives and may not be reliable. Sometimes it was difficult to assess the type of chemical or drug agents used for self-poisoning owning to the selfreporting history from patients or relatives. However, despite these limitations findings of this study will hopefully help target prevention and intervention efforts for those most at risk of selfinflicted injuries in this region. The challenges identified in the management of these patients in our setting need to be addressed, in order to deliver optimal trauma care for the victims of selfinflicted injuries.

In conclusion self-inflicted injuries are an emerging but neglected public health problem in our environment and have become a major cause of unacceptably high morbidity and mortality among young males in our society where resources for prehospital and hospital trauma care are limited. Psychiatric illnesses, poverty, family disharmony, loss of employment, substance abuse, chronic illness and poor education have been reported to be responsible for these injuries in our society. Addressing the root causes of these motivating factors will reduce the incidence of selfinflicted injuries in our environment. Establishing efficient emergency health care services for prehospital care and effective ambulance system for rapid transport of injured victims to hospital will reduce morbidity and mortality associated with these injuries.

\section{Acknowledgements}

The authors thank all members of staff of Department of Surgery who participated in the preparation of this manuscript, and all those who were involved in the care of our patients. Special thanks go to members of the Medical record department for their assistance in the retrieval of patients' case notes.

\section{References}

Adoga, A.A., D Ma'an, N., Embu, H.Y. \& Obindo, T.J. (2010) Management of suicidal cut throat injuries in a developing nation: three case reports. Cases Journal 3: 65.

Bailey, A.R. (1997) Management of a patient with a cut throat. British Journal of Hospital Medicine 58: 469

Bhattacharjee, N., Arefin, S.M., Mazumder, S.M. \& Khan, M.K. (1997) Cut throat injury: a retrospective study of 26 cases. Bangladesh Medical Research Council Bull 23:87-90

Corso, P.S., Mercy, J.A., Simon, T.R., Finkelstein, E.A. \& Miller, T.R. (2007) Medical Costs and Productivity Losses Due to Interpersonal Violence and Self- Directed Violence. American Journal of Preventive Medicine 32: 474-482.

Demyttenaere, S.V., Nansamba, C., Nganwa, A., Mutto, M., Lett, R. \& Razek, T. (2009) Injury in Kampala, Uganda: 6 years later. Canadian Journal of Surgery 52: 146-150.

Doshi, A., Boudreaux, E.D., Wang, N., Pelletier, A.J., Camargo, C.A. Jr. (2005) National study of US emergency department visits for attempted suicide and self-inflicted injury, 1997-2001. Annals of Emergency Medicine 46: 369-375.

Duncan, J.A.T. (1975) A case of severely cut throat. British Journal of Anesthesia 47: 1327-1329.

Dyck, R.J., Bland, R.C., Newman, S.C. \& Orn, H. (1988) Suicide attempts and psychiatric disorders in Edmonton. Acta Psychiatrica Scandinavia 338: 64-71.

Gilyoma, J.M., Chalya, P.L. (2014) Cut throat injuries at a university teaching hospital in northwestern Tanzania: a review of 98 cases. BMC Emergency Medicine 14: 1.

Goldney, R.D. (2002) A global view of suicidal behaviour. Emergency Medicine 14: 24-34.

Greydanus, D. \& Shek, D. (2009) Deliberate self-harm and suicide in adolescents. The Keio Journal of Medicine 58:144-151 
Herzog, M., Hoppe, F., Baier, G. \& Dieler, R. (2005) Injuries of the head and neck in suicidal intention. Laryngorhinootologie 84: 176-181.

Karch, D., Dahlberg, L. \& Patel, N. (2010) Surveillance for Violent Deaths-National Violent Death Reporting System, 16 states, 2006. MMWR 59: 1-50.

Kobusingye, O.C. (2008) Violence and Injuries: What Africa should do? African Health Monitor 3740.

Ladapo, A.A. (1979) Open injuries of the anterior neck. Ghana Medical Journal 18: 182-186.

Lopez, A.D., Mathers, C.D., Ezzati, M., Jamison, D.T. \& Murray, C.J. (2006) Global and regional burden of disease and risk factors, 2001: systematic analysis of population health data. Lancet 367: 17470-174757.

Manilal, A., Khorshed, A.B.M., Talukder, D.C., Sarder, R.M.A., Fakir, A.T. \& Hossain, M. (2011) Cut throat injury: review of 67 cases. Bangladesh Journal of Otorhinolaryngology 17: 5-13.

Marbella, A.M., Yang, H., Guse, C.E., Meurer, J.R. \& Layde, P.M. (2005) Adolescent hospital discharges associated with self-poisonings in Wisconsin, 2000-2002. Wisconsin Medical Journal 104: 59-64.

Mathers, C.D., Boerma, T. \& Fat, D. (2009) Global and regional causes of death. British Medical Bulletin 9: 7-32.

Mathers, C.D. \& Loncar, D. (2006) Projections of global mortality and burden of disease from 2002 to 2030. PloS Medicine 3: e422

Moshiro, C., Ivar, H., Anne, N., Philip, S., Yusuf, H. \& Gunnar, K. (2005) Injury morbidity in an urban and a rural area in Tanzania: an epidemiological survey. BMC Public Health 5: 11.

Mutooro, S.M., Mutakooha, E. \& Kyamanywa, P. (2010) A comparison of Kampala trauma score II with the new injury severity score in Mbarara University Teaching Hospital in Uganda. East Central African Journal of Surgery 15: 62-70.

Norberg, E. (2000) Injuries as a public health problem in sub-Saharan Africa: epidemiology and prospects for control. East African Medical Journal 77:S1-S43.

Olfson, M., Gameroff, M.J., Marcus, S.C., Greenberg, T. \& Shaffer, D. (2006) Emergency treatment of young people following deliberate self-harm. Archives of General Psychiatry 62: $1122-1128$.

Platt, S. (1984) Unemployment and suicidal behavior: a review of the literature. Social Science \& Medicine 19: 93-115.

Reith, D.M., Whyte, I., Carter, G. \& McPherson, M. (2003) Adolescent self-poisoning: a cohort study of subsequent suicide and premature deaths. Crisis 24: 79-84.

Shah, A. \& Bhandarkar, R. (2008) Cross-national study of the correlation of general population suicide rates with unemployment rates. Psychology of Reproduction 103: 793-796.

Spicer, R.S. \& Miller, T.R. (2000) Suicide acts in 8 states: incidence and case fatality rates by demographics and method. American Journal of Public Health 90:1885-1891.

Suominen. K., Henriksson, M., Suokas, J., Isometsa, E., Ostamo, A. \& Lonnqvist, J. (1996) Mental disorders and comorbidity in attempted suicide. Acta Psychiatrica Scandinavia 94: 234-240.

Vajani, M., Annest, J.L., Crosby, A.E., Alexander, J.D. \& Millet, L.M. (2007) Nonfatal and fatal selfharm injuries among children aged 10-14 years--United States and Oregon, 2001-2003. Suicide and Life Threatening Behaviour 37: 493-506. 